\title{
Isomorphism for Graphs of Bounded Distance Width*
}

\author{
Koichi Yamazaki, Hans L. Bodlaender, Babette de Fluiter, Dimitrios M. Thilikos \\ Department of Computer Science, Utrecht University, \\ P.O. Box 80.089, 3508 TB Utrecht, the Netherlands \\ E-mail: \{koichi,hansb,babette,sedthilk\}@cs.ruu.nl
}

\begin{abstract}
In this paper, we study the Graph Isomorphism problem on graphs of bounded treewidth, bounded degree or bounded bandwidth. Graph Isomorphism can be solved in polynomial time for graphs of bounded treewidth, pathwidth, or bandwidth, but the exponent depends on the treewidth, pathwidth, or bandwidth. Thus, we look for special cases where 'fixed parameter tractable' polynomial time algorithms can be established. We introduce some new and natural graph parameters: the (rooted) path distance width, which is a restriction of bandwidth, and the (rooted) tree distance width, which is a restriction of treewidth. We give algorithms that solve Graph Isomorphism in $O\left(n^{2}\right)$ time for graphs with bounded rooted path distance width, and in $O\left(n^{3}\right)$ time for graphs with bounded rooted tree distance width. Additionally, we show that computing the path distance width of a graph is NP-hard, but both path and tree distance width can be computed in $O\left(n^{k+1}\right)$ time, when they are bounded by a constant $k$; the rooted path or tree distance width can be computed in $O(n e)$ time. Finally, we study the relationships between the newly introduced parameters and other existing graph parameters.
\end{abstract}

\section{Introduction}

In this paper, we consider the Graph Isomorphism problem, for graphs for which certain parameters can be assumed to be small constants. We are especially interested in Graph Isomorphism on graphs where either the treewidth, bandwidth, or degree of the graph is bounded, as there are many interesting graph classes which have a bound on one of these parameters (see e.g. [2]). For these parameters, it has been shown that when the parameter is a constant, then Graph Isomorphism is polynomial time solvable (see [12] for bounded degree, and [3] for bounded treewidth and bandwidth). However, in each of these three cases, the exponent of the algorithm grows with the parameter. Thus, a question is, whether algorithms exist for Graph Isomorphism with a running time

\footnotetext{
${ }^{*}$ The first author was supported by Ministry of Education, Science, Sports and Culture of Japan as Oversea's Research Scholar. The second and last author were partially supported by ESPRIT Long Term Research Project 20244 (project ALCOM IT: Algorithms and Complexity in Information Technology). The third author was supported by the Foundation for Computer Science (S.I.O.N) of the Netherlands Organisation for Scientific Research (N.W.O.). The last author was supported by the Training and Mobility of Researchers (TMR) Program, (EU contract no ERBFMBICT950198).
} 
$O\left(f(k) n^{c}\right), c$ a small constant, $k$ the maximum degree / treewidth / bandwidth / . . / of the graph; in other words, whether Graph Isomorphism is fixed parameter tractable (in the sense of the fixed parameter complexity theory of Downey and Fellows, see e.g. $[8,9])$, with the maximum degree / treewidth / bandwidth / . . as parameter.

Thus, we are looking for answers to the questions whether Graph Isomorphism is fixed parameter tractable for the case that the parameter is the degree, treewidth or bandwidth of the graph. These questions are apparently hard. In this paper, we are able to solve some interesting special cases of these problems.

For this, several natural graph parameters are introduced: the (rooted) path distance width, and the (rooted) tree distance width. The notion of path distance width can be seen to have a close relation to bandwidth; the notion of tree distance width is a natural tree-like generalisation of this notion, with a close relationship to treewidth.

This paper is organised as follows. In section 2 we prove that the rooted path (tree) distance width of a graph can be computed in $O(n e)$ time, that computing the path distance width of a graph is NP-hard, but if the path or tree distance width is at most some fixed constant $k$, then the minimum path (tree) distance width can be computed in $O\left(n^{k+1}\right)$ time. The main results of the paper can be found in Section 3: it is shown that Graph Isomorphism is solvable in $O\left(n^{2}\right)$ time for graphs with bounded rooted path distance width, thus solving a significant special case of Graph Isomorphism for graphs of bounded bandwidth. Furthermore, it is shown that Graph Isomorphism is solvable in $O\left(n^{3}\right)$ time for graphs with bounded rooted tree distance width, which solves a special case for Graph Isomorphism for graphs of bounded treewidth. In Section 4, the relations between the different considered parameters are investigated.

\section{Definitions and Complexity Results for Distance Width}

The graphs we consider are simple, undirected and connected, and contain no self loops. For a graph $G$, we denote its set of vertices by $V(G)$ and its set of edges by $E(G)$. Also, we denote as $G[S]$ the subgraph of $G$ induced by $S \subseteq V(G)$. For two graphs $G$ and $H$, a function $f: V(G) \rightarrow V(H)$ is called an isomorphism (from $G$ to $H$ ) if $f$ is a bijection and for each $v, w \in V(G),\{v, w\} \in E(G)$ iff $\{f(v), f(w)\} \in E(H)$. Two graphs $G$ and $H$ are isomorphic if there is an isomorphism from $G$ to $H$. The Graph Isomorphism problem is the problem of checking for two given graphs whether they are isomorphic.

A graph parameter is a function which maps each graph to a positive integer.

We first review a number of graph parameters.

- A tree decomposition of a graph $G=(V, E)$ is a pair $\left(\left\{X_{i} \mid i \in I\right\}, T=(I, F)\right)$, where $\left\{X_{i} \mid i \in I\right\}$ is a collection of subsets of $V$ and $T$ is a tree, such that

$-\bigcup_{i \in I} X_{i}=V(G)$,

- for each edge $\{v, w\} \in E$, there is an $i \in I$ such that $v, w \in X_{i}$, and

- for each $v \in V$ the set of nodes $\left\{i \mid v \in X_{i}\right\}$ forms a subtree of $\mathrm{T}$.

The width of a tree decomposition $\left(\left\{X_{i} \mid i \in I\right\}, T=(I, F)\right)$ equals $\max _{i \in I}\left(\left|X_{i}\right|-1\right)$. The treewidth of a graph $G$ is the minimum width over all tree decompositions 
of $G$. The corresponding graph parameter (which is the function that maps each graph to its treewidth) is denoted by $\mathcal{T} \mathcal{W}$.

A (linear) layout of a graph $G$ is a one-to-one function $f: V(G) \rightarrow\{1, \ldots,|V(G)|\}$.

- The bandwidth of a layout $f$ of a graph $G$ is defined as $\max _{\{u, v\} \in E(G)}|f(u)-f(v)|$. The bandwidth of a graph $G$ is the minimum bandwidth over all layouts of $G$. The corresponding graph parameter is denoted by $\mathcal{B W}$.

For a given graph $G$ and two vertices $u, v \in V(G), d_{G}(u, v)$ denotes the distance between $u$ and $v$, which is the number of edges on a shortest path between $u$ and $v$. For a set $S \subseteq V(G)$ and a vertex $w \in V(G), d_{G}(S, w)$ denotes $\min _{v \in S} d_{G}(v, w)$.

- A tree distance decomposition of a graph $G=(V, E)$ is a triple $\left(\left\{X_{i} \mid i \in I\right\}, T=\right.$ $(I, F), r)$, where

$-\bigcup_{i \in I} X_{i}=V(G)$ and for all $i \neq j, X_{i} \cap X_{j}=\emptyset$,

- for each $v \in V$, if $v \in X_{i}$, then $d_{G}\left(X_{r}, v\right)=d_{T}(r, i)$, and

- for each edge $\{v, w\} \in E$, there are $i, j \in I$ such that $v \in X_{i}, w \in X_{j}$ and either $i=j$ or $\{i, j\} \in F$.

Node $r$ is called the root of the tree $T$, and $X_{r}$ is called the root set of the tree distance decomposition. The width of a tree distance decomposition $\left(\left\{X_{i} \mid i \in\right.\right.$ $I\}, T, r)$ is equal to $\max _{i \in I}\left|X_{i}\right|$. The tree distance width of a graph $G$ is the minimum width over all possible tree distance decompositions of $G$. The corresponding graph parameter is denoted by $\mathcal{T} \mathcal{D} \mathcal{W}$.

- A rooted tree distance decomposition of a graph $G=(V, E)$ is a tree distance decomposition $\left(\left\{X_{i} \mid i \in I\right\}, T=(I, F), r\right)$ of $G$ in which $\left|X_{r}\right|=1$. The rooted tree distance width of a graph $G$ is the minimum width over all rooted tree distance decompositions. The corresponding graph parameter is denoted by $\mathcal{R} \mathcal{T} \mathcal{D W}$.

- The (rooted) path distance decomposition and the parameter of (rooted) path distance width of a graph $G=(V, E)$ are defined similar to the (rooted) tree distance decomposition and (rooted) tree distance width, but now the tree $T$ is required to be a path. For reasons of simplicity we will denote a (rooted) path distance decomposition as $\left(X_{1}, X_{2}, \ldots, X_{t}\right)$, where $X_{1}$ is the root set of the decomposition. We denote the corresponding graph parameters by $\mathcal{P} \mathcal{D W}$, and $\mathcal{R} \mathcal{P} \mathcal{D} \mathcal{W}$, respectively.

It is easy to check that for any graph $G, \mathcal{T W}(G) \leq 2 \mathcal{T} \mathcal{D W}(G)-1, \mathcal{T} \mathcal{D W}(G) \leq$ $\mathcal{R} \mathcal{T} \mathcal{D W}(G), \mathcal{B W}(G) \leq 2 \mathcal{P} \mathcal{D W}(G)-1$ and $\mathcal{P} \mathcal{D W}(G) \leq \mathcal{R} \mathcal{P} \mathcal{D W}(G)$. Hence fixed parameter tractability of Graph Isomorphism for $\mathcal{T W}$ implies the same for $\mathcal{T D W}$ and $\mathcal{R} \mathcal{T} \mathcal{W}$, and fixed parameter tractability of Graph Isomorphism for $\mathcal{B} \mathcal{W}$ implies the same for $\mathcal{P} \mathcal{D W}$ and $\mathcal{R} \mathcal{P} \mathcal{D W}$. Also, showing that for Graph Isomorphism is fixed parameter tractable for e.g. $\mathcal{R} \mathcal{P} \mathcal{D W}$ might give more insight in whether it is fixed parameter tractable for $\mathcal{B W}$. Therefore, we study the complexity of Graph Isomorphism on graphs for which $\mathcal{P} \mathcal{D W}, \mathcal{R} \mathcal{P} \mathcal{D W}, \mathcal{T} \mathcal{D W}$ or $\mathcal{R} \mathcal{T} \mathcal{D W}$ is bounded. 


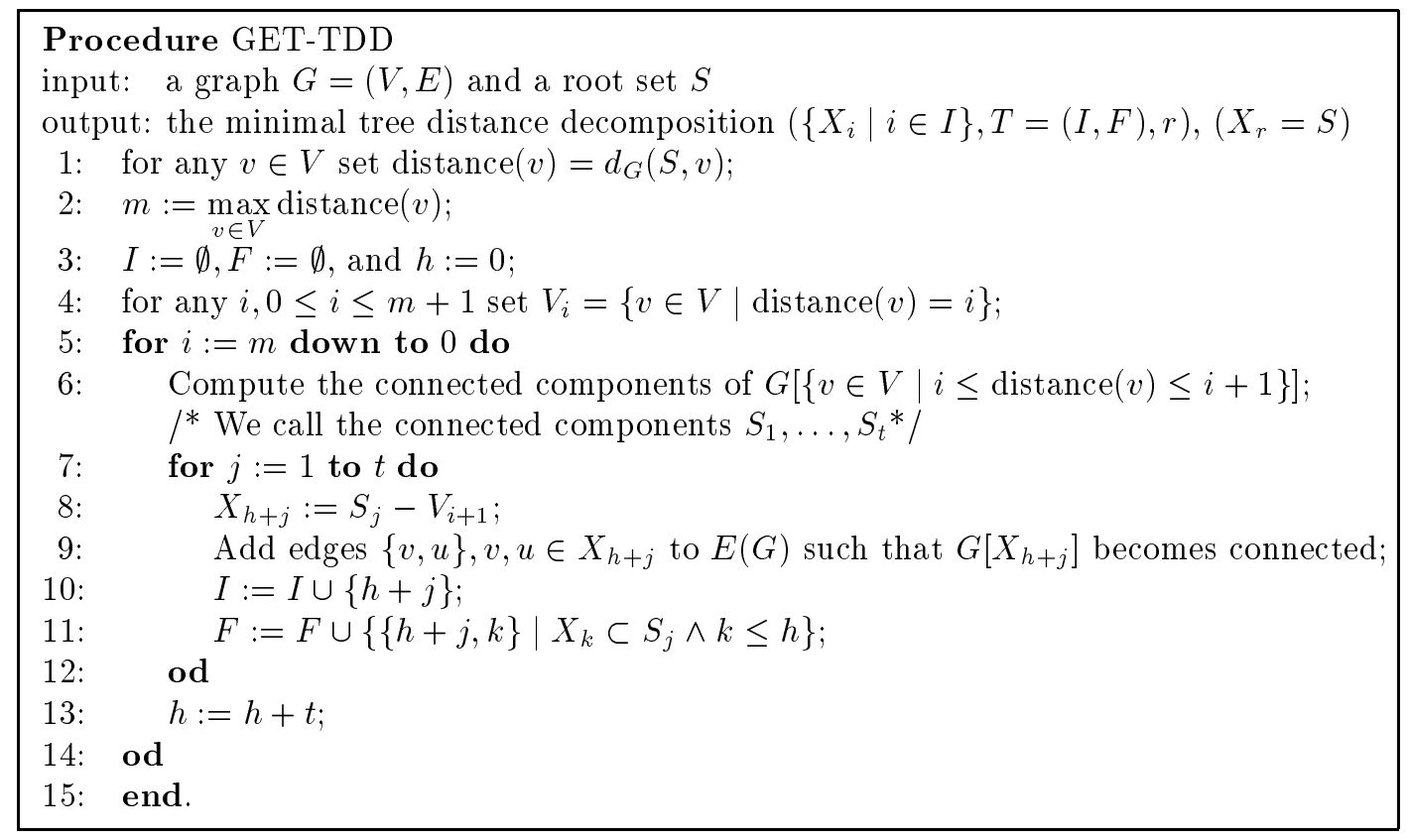

Figure 1: Procedure GET-TDD $(G, S)$.

For a given graph $G$ and $S \subseteq V(G)$, there is a unique path distance decomposition of $G$ with root set $S$, and this decomposition can be found in $O(e)$ time $(e=|E(G)|)$ : for each vertex $v \in V(G)$, compute $d_{G}(S, v)$ using breadth first search. Then for each possible distance $d$, make a node $X_{d}$ containing all vertices with distance $d$ to $S$.

Definition Let $D=\left(\left\{X_{i} \mid i \in I\right\}, T=(I, F), r\right)$ be a tree distance decomposition of $G$. Given a vertex $v \in I$ we denote as $T_{v}$ the connected subtree of $T$ induced by all the nodes in $I$ that are connected with $r$ via paths containing $v$. Finally, given a node $i \in I$ we set $V(D, i)=\bigcup_{w \in V\left(T_{i}\right)} X_{w}$.

For a given graph $G$ and set $S \subseteq V(G)$, there may be more than one tree distance decomposition with root set $S$. However we define the minimal tree distance decomposition of a graph $G$ with root set $S$ as follows.

Definition Let $D=\left(\left\{X_{i} \mid i \in I\right\}, T=(I, F), r\right)$ be a tree distance decomposition of $G$. We call $D$ minimal if, for each $i \in I, G[V(D, i)]$ is connected.

An immediate consequence of the definition above is that given a graph $G$ and a root set $S$ the minimal tree distance decomposition is uniquely defined. Also, it can be found with procedure GET-TDD presented in Figure 1, which can be made to run in $O(e)$ time.

Theorem 2.1 Given a graph $G$ and a set $S \subseteq V(G)$, we can compute in $O(|E(G)|)$ time the unique path distance decomposition with root set $S$, or the unique minimal tree distance decomposition with root set $S$. 
Proof. Let $D=\left(\left\{X_{i} \mid i \in I\right\}, T=(I, F), r\right)$ be some output of GET-TDD. It is easy to see that $D$ is a tree distance decomposition of $G$.

We will prove that for any $i \in I, G[V(D, i)]$ is connected. Let $m=\max _{i \in I} d_{T}(r, i)$. We will use induction on $m-d_{T}(r, i)$. If $d_{T}(r, i)=m$, then it is clear from the algorithm that $G[V(D, i)]$ is connected.

Assume that for any $i \in I$ such that $d_{T}(r, i)>n(0<n \leq m), G[V(D, i)]$ is connected. Let $i \in I$ such that $d_{T}(r, i)=n$. Let also $\left\{i_{1}, \ldots, i_{t}\right\} \subseteq I$ be such that for all $i_{\sigma}, 1 \leq \sigma \leq t, d_{T}\left(r, i_{\sigma}\right)=n+1$ and $\left\{\left\{i, i_{1}\right\}, \ldots,\left\{i, i_{t}\right\}\right\} \subseteq F$. We will prove that $G[V(D, i)]$ is connected, that is, we will show that for any pair of vertices $v, u \in V(D, i)$, there exists a path connecting $u$ and $v$ in $G[V(D, i)]$. From the induction hypothesis and the fact that all vertices in $L_{n+1}=X_{i_{1}} \cup \ldots \cup X_{i_{t}}$ are adjacent to a vertex in $X_{i}$, we have that there exist two vertices $v^{\prime}, u^{\prime}$ in $X_{i}$ connected by some paths in $G[V(D, i)]$ with $v$ and $u$ respectively. The connectivity of $G[V(D, i)]$ follows now easily in the case where $G\left[X_{i} \cup L_{n+1}\right]$ is connected. Suppose that $G\left[X_{i} \cup L_{n+1}\right]$ is not connected. In this case we notice that $X_{i} \cup L_{n+1}$ must induce one of the connected components computed during the $(m-n+1)$ th execution of step 5 ( $m$ is defined at step 2$)$. Clearly, each of these connected components became connected because of the addition of some number of edges connecting vertices in $X_{i_{\sigma}}, 1 \leq \sigma \leq t$ during the $(m-n)$ th execution of loop $7-12$. We call these edges new edges and denote the current graph after the $(n-1)$ th loop by $G_{n}$. As there exists in $G_{n}\left[X_{i} \cup L_{n+1}\right]$ a path connecting $v^{\prime}$ and $u^{\prime}$, such a path must contain a number of new edges (see Figure 2). From the induction hypothesis we have that any new edge corresponds to a path in $G\left[V\left(D, i_{\sigma}\right)\right]$ connecting its endpoints for some $\sigma, 1 \leq \sigma \leq t$. Thus, we can construct a path in $G[V(D, i)]$ connecting $u^{\prime}$ and $v^{\prime}$. Therefore, there exists a walk (hence path) connecting $u$ and $v$ and the connectivity of $G[V(D, i)]$ follows.

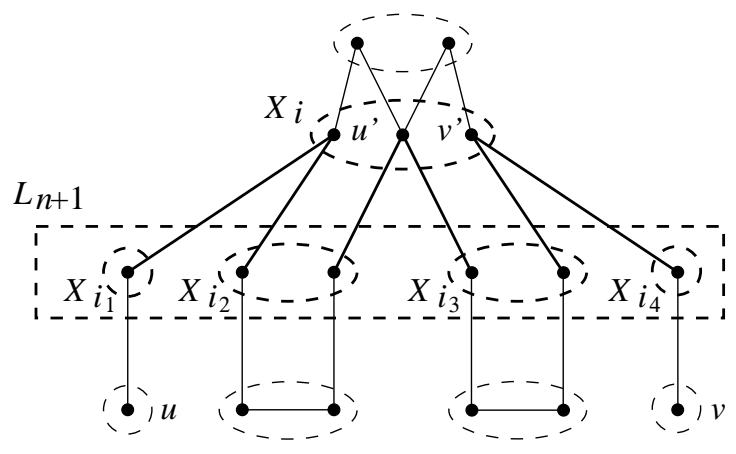

The original graph

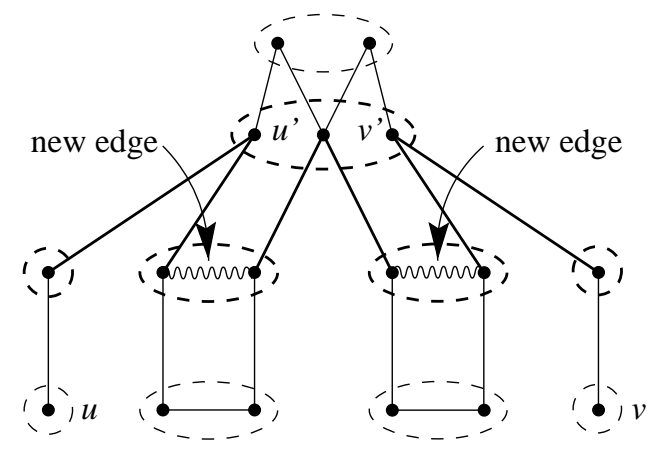

The graph after the 2nd execution of the loop defined by steps 6-13 in GET-TDD

Figure 2: An example for the procedure GET-TDD.

Step 1 uses breadth first search to assign for each vertex in the graph its distance from the root set. This costs $O(|E(G)|)$ time. Also loop 5-14 needs in total $O(|E(G)|)$ time as the executions of step 6 use in total $O(|E(G)|)$ time and each call of line 9 can be executed in $O\left(\left|V\left(X_{h+j}\right)\right|\right)$ time. 
From Theorem 2.1 and the fact that, if a graph has (rooted) tree or path distance width at most $k$, then $e=O(k n)$, we get the following result.

Corollary 2.2 There is an algorithm that computes a rooted path (tree) distance decomposition of minimum width of a graph $G$ in $O\left(k n^{2}\right)$ time, where $k$ is the rooted path (tree) distance width of the graph.

There is an algorithm that computes a path (tree) distance decomposition of minimum width of a graph in $O\left(k n^{k+1}\right)$ time, where $k$ denotes the path (tree) distance width of the graph.

The following result concerns the complexity of (rooted) path (tree) distance width.

Theorem 2.3 The following problem is NP-complete even if the input graphs are trees. Given a graph $G$ and an integer $k$, does $G$ have path distance width at most $k$ ?

Proof. The proof is based on a reduction from the following strongly NP-complete problem:

3PARTITION

Instance: A set $A=\left\{a_{1}, \ldots, a_{3 m}\right\}$ of positive integers and an integer value $B$ such that $B / 4<a_{i}<B / 2,1 \leq i \leq 3 m, m>1$, and $\sum_{1 \leq i \leq 3 m} a_{i}=m B$.

Question: Is there a partition of $A$ into $m$ disjoint sets $A_{1}, A_{2}, \cdots, A_{m}$ such that $\sum_{a \in A_{i}} a=B, 1 \leq i \leq m$ ?

We describe a transformation that, given an instance of the 3PARTITION problem, outputs a tree $T$ such that $T$ has path distance width at most $d$ iff the answer for the 3PARTITION problem is yes.

Let $c=57 m+1$ and $d=c \cdot B+9 m$. T is constructed as follows: First we set $U_{i}=\left\{u_{i, 1}, \ldots, u_{i, m}\right\}, V_{i}=\left\{v_{i, 1}, \ldots, v_{i, c a_{i}}\right\}, 1 \leq i \leq 3 m$, and $W=\left\{w_{1}, \ldots, w_{2 d-12 m}\right\}$. Then, we define $T_{i}=\left(\{x\} \cup U_{i} \cup V_{i},\left\{\left\{x, u_{i, 1}\right\}\right\} \cup\left\{\left\{u_{i, j}, u_{i, j+1}\right\} \mid 1 \leq j \leq m-1\right\} \cup\right.$ $\left.\left\{\left\{v_{i, j}, u_{i, m}\right\} \mid 1 \leq j \leq c \cdot a_{i}\right\}\right), 1 \leq i \leq 3 m$. Now, set $T=\left(W \cup \bigcup_{1<i<3 m} V\left(T_{i}\right),\left\{\left\{x, w_{i}\right\} \mid\right.\right.$ $\left.1 \leq i \leq 2 d-12 m\} \cup\left(\bigcup_{1 \leq i \leq 3 m} E\left(T_{i}\right)\right)\right)$ (see also Figure 3 ).

We will prove now that the 3 PARTITION instance has a solution iff $T$ has a path distance decomposition $\left(X_{1}, X_{2}, \ldots, X_{t}\right)$ with width at most $d$.

Let $\left\{A_{1}, A_{2}, \cdots, A_{m}\right\}$ be a solution of the 3PARTITION problem. We construct a path distance decomposition as follows. We first define $f:\{1,2, \ldots, 3 m\} \mapsto\{x\} \cup \underset{1 \leq i \leq 3 m}{\bigcup} U_{i}$ such that $f(i)=x$ iff $a_{i} \in A_{1}$ and $f(i)=u_{i, j-1}$ iff $a_{i} \in A_{j}, 2 \leq j \leq m$. We set $S=\left\{w_{1}, w_{2}, \cdots, w_{d-3 m}\right\} \cup\{f(1), \ldots, f(3 m)\}$. Let $\left(X_{1}=S, X_{2}, \ldots, X_{r}\right)$ be the path distance decomposition of $G$ with root set $S$.

Now we prove that for all $i, 1 \leq i \leq r,\left|X_{i}\right| \leq d$. By the construction, we have that the cardinality of the root set $S=X_{1}$ is no more than $d$. We also observe that $X_{2}$ contains $d-9 m$ vertices from $W$, and at most $3 m+2 \cdot 3 m$ vertices from $U_{T}=\{x\} \cup \bigcup_{1 \leq i \leq 3 m} U_{i}$. So $\left|X_{2}\right| \leq d$. It is now easy to see that for any $i>2, X_{i}$ must contain at most $3 \cdot 3 m$ vertices 


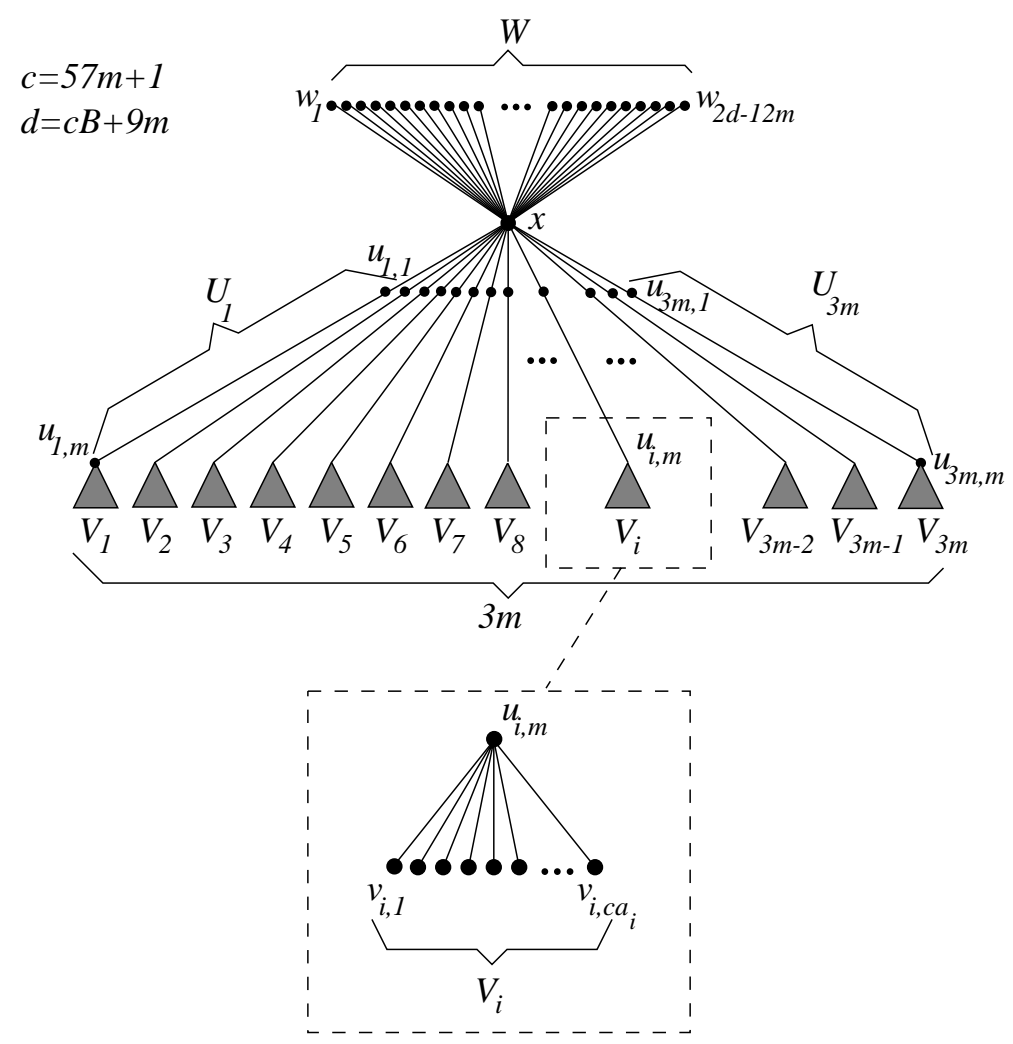

Figure 3: The reduction of Theorem 2.3.

from $U_{T}$ and $c a_{i_{1}}+c a_{i_{2}}+c a_{i_{3}}$ vertices from $V_{i_{1}} \cup V_{i_{2}} \cup V_{i_{3}}$ where $A_{j}=\left\{a_{i_{1}}, a_{i_{2}}, a_{i_{l}}\right\}$ is some set $A_{j}$ of the solution of the 3PARTITION problem. Thus $c a_{i_{1}}+c a_{i_{2}}+c a_{i_{3}}=c B$ and for any $i>2, X_{i}$ must contain at most $d=c B+9 m$ vertices. Therefore, $\left(X_{1}, \ldots, X_{r}\right)$ has distance width at most $d$.

Suppose now that $\left(X_{1}, \ldots, X_{t}\right)$ is a path distance decomposition of $T$ that has width at most $d$. We prove that there exists a partition of $A=\left\{a_{1}, \ldots, a_{3 m}\right\}$ into $m$ disjoint sets $A_{1}, \ldots, A_{m}$ such that $\sum_{a \in A_{i}} a=B, 1 \leq i \leq m$. We distinguish two cases:

Case 1: $x \in X_{1}$. In this case we easily observe that $W \subseteq X_{1} \cup X_{2}$ and thus we have $\left|X_{1} \cap W\right|+\left|X_{2} \cap W\right|=|W|=2 d-12 m$. Using this and the fact that $\left|X_{1}\right|,\left|X_{2}\right| \leq d$, we have that $2 d \geq\left|X_{1}\right|+\left|X_{2}\right|=\left|X_{1}-W\right|+\left|X_{1} \cap W\right|+\left|X_{2}-W\right|+\left|X_{2} \cap W\right|=$ $\left|X_{1}-W\right|+\left|X_{2}-W\right|+2 d-12 m \geq\left|X_{i}-W\right|+2 d-12 m \Rightarrow\left|X_{i}-W\right| \leq 12 m, i=1,2$.

Let $\left\{R_{j} \mid j \geq 1\right\}$ be a collection of subsets of $\{1,2, \ldots, 3 m\}$ such that $i \in R_{j}$ iff $\left|X_{j} \cap V_{i}\right| \geq c a_{i}-12 m$.

We first claim that for all $i, 1 \leq i \leq 3 \mathrm{~m}$, there exists at most one $j \geq 1$ such that $i \in R_{j}$ Suppose that for some $i$, there are $j$ and $j^{\prime}$ such that $j \neq j^{\prime}, i \in R_{j}$ and $i \in R_{j^{\prime}}$. In such a case we have that $\left|X_{j} \cap V_{i}\right| \geq c a_{i}-12 m$ and $\left|X_{j^{\prime}} \cap V_{i}\right| \geq c a_{i}-12 m$. As $X_{j} \cap X_{j^{\prime}}=\emptyset$, we conclude that $\left|V_{i}\right| \geq 2 c a_{i}-24 m \Rightarrow c a_{i} \geq 2 c a_{i}-24 m \Rightarrow 24 m \geq c a_{i} \geq c=57 m+1$, a contradiction.

We now claim that for all $i, 1 \leq i \leq 3 m$, there exists a $j \geq 1$, such that $i \in R_{j}$. 
Choose $j^{\prime}$ such that $u_{i, m} \in X_{j^{\prime}}$. Notice that for all $v \in V_{i}$, either $v \in X_{1}$ or $v \in X_{j^{\prime}+1}$. As $X_{1} \cap V_{i} \subseteq X_{1}-W$, we have that $\left|X_{1} \cap V_{i}\right| \leq 12 m$ and thus $\left|X_{j^{\prime}+1} \cap V_{i}\right| \geq c a_{i}-12 m \Rightarrow$ $i \in R_{j^{\prime}+1}$.

We claim that if $j=1,2$ then $R_{j}=\emptyset$. Indeed, suppose, on the contrary, that for some $j=1,2$ there exists an $i, 1 \leq i \leq 3 \mathrm{~m}$ such that $i \in R_{j}$. In this case we have that $\left|X_{j} \cap V_{i}\right| \geq c a_{i}-12 m$. But, as $\left|X_{j}-W\right| \leq 12 m, j=1,2$ and $X_{j} \cap V_{i} \subseteq X_{j}-W, j=$ $1,2,1 \leq i \leq 3 m$, we conclude that $12 m \geq\left|X_{j}-W\right| \geq\left|X_{j} \cap V_{i}\right| \geq c a_{i}-12 m \geq c-12 m=$ $57 m+1-12 m=45 m+1$, a contradiction. Notice also that, as $x \in X_{1}$, we have that $V(T) \subseteq X_{1} \cup \cdots \cup X_{m+2}$ and thus for all $j \geq m+3, R_{j}=\emptyset$. A.

We now set $A_{j}=\left\{a_{i} \mid i \in R_{j+2}\right\}, 1 \leq j \leq m$. Clearly, $A_{1}, \ldots, A_{m}$ is a partition of

We claim that for all $j, 1 \leq j \leq m,\left|A_{j}\right| \leq 3$. Suppose, on the contrary that for some $j, 1 \leq j \leq m,\left|A_{j}\right| \geq 4$. Then $\sum_{a_{i} \in A_{j}}\left|X_{j+2} \cap V_{i}\right| \geq \sum_{a_{i} \in A_{j}}\left(c a_{i}-12 m\right) \geq$ $\sum_{a_{i} \in A_{j}}\left(c \frac{B+1}{4}-12 m\right) \geq 4\left(\frac{c B}{4}+\frac{c}{4}-12 m\right) \geq c B+c-48 m \geq c B+9 m+1>d$, a contradiction.

From the claims above, we conclude that the sets $A_{1}, \ldots, A_{m}$ form a partition of $A$ into sets consisting of at most 3 elements.

What now remains to be proven is that for all $j, 1 \leq j \leq m, \sum_{a \in A_{j}} a=B$. Clearly, as $\sum_{1 \leq i \leq 3 m} a_{i}=m B$, it is sufficient to prove that for all $j, 1 \leq j \leq m, \sum_{a \in A_{j}} a \leq B$. For this, we first notice that $c \sum_{a_{i} \in A_{j}} a_{i} \leq \sum_{a_{i} \in A_{j}}\left(\left|X_{j+2} \cap V_{i}\right|+12 m\right) \leq\left|X_{j+2}\right|+36 m \leq$ $d+36 m=c B+9 m+36 m \leq c B+45 m$. Finally, we have that $\sum_{a_{i} \in A_{j}} a_{i} \leq B+\frac{45 m}{c}$ which implies $\sum_{a_{i} \in A_{j}} a_{i} \leq B+\left\lfloor\frac{45 m}{c}\right\rfloor=B\left(\left\lfloor\frac{45 m}{c}\right\rfloor=0\right.$ because $\left.c=57 m+1>45 m\right)$.

Case 2: $x \notin X_{1}$. In this case we can easily see that $X_{1} \cup W \neq \emptyset$ because otherwise, if $x \in X_{i}$ for some $i$, then $W \subseteq X_{i+1}$, but $|W|>d$. So, $x \in X_{2}$, and $W \subseteq X_{1} \cup X_{3} \Rightarrow$ $\left|X_{1} \cap W\right|+\left|X_{3} \cap W\right|=2 d-12 m$. Using the fact that $\left|X_{1}\right|,\left|X_{3}\right| \leq d$ and, using a similar argument as in Case 1, we have that $\left|X_{i}-W\right| \leq 12 m, i=1,3$.

We define $\left\{R_{j} \mid j \geq 1\right\}$ as in Case 1 and following a similar argumentation, we deduce that any $i \in\{1, \ldots, 3 m\}$ belongs to exactly one $A_{j}$. Also very similarly to Case 1 we have that if $i=1$ or 3 , then $R_{j}=\emptyset$. Moreover, we claim that $R_{m+2}=\emptyset$. Suppose, on the contrary, that $i \in R_{m+2}$. This means that $\left|X_{m+2} \cap V_{i}\right| \geq c a_{i}-12 m$ and thus there exists some vertex $w \in X_{m+2}$ that belongs in $V_{i}$. Notice now that, as $d_{T}\left(X_{1}, w\right)=m+1 \geq 3$ we have that for all $h, 1 \leq h \leq m, u_{i, h} \in X_{h+1}$ and thus $x \in X_{1}$, contradiction. (if $x \in X_{i}, i>2$ then $W \subseteq X_{i+1}$ a contradiction) Finally, as $x \in X_{2}$, and for all $w \in V(T), d_{T}(x, w) \leq m+1$, we easily conclude that for all $j \geq m+4$, $X_{j}=\emptyset \Rightarrow R_{j}=\emptyset$.

We set $A_{1}=\left\{a_{i} \mid i \in R_{2}\right\}, A_{j}=\left\{a_{j} \mid i \in R_{j+2}\right\}, 2 \leq j \leq m-1$ and $A_{m}=\left\{a_{i} \mid i \in R_{m+3}\right\}$. Following now the same steps as in Case 1, one can prove that $A_{1}, \ldots, A_{m}$ is a partition of $A$ into sets consisting of at most 3 elements such that for all $j, 1 \leq j \leq m, \sum_{a \in A_{j}} a=B$. 


\section{Graph Isomorphism for Graphs of Bounded Distance Width}

In this section, it is shown that the Graph Isomorphism problem is fixed parameter tractable for graphs of bounded rooted path distance width or bounded rooted tree distance width.

We present an algorithm with running time $O\left(n^{2}\right)$ that tests isomorphism for two graphs with rooted path distance width at most some constant $k$, and an algorithm with running time $O\left(n^{3}\right)$ that tests isomorphism for graphs with rooted tree distance width at most some constant $k$.

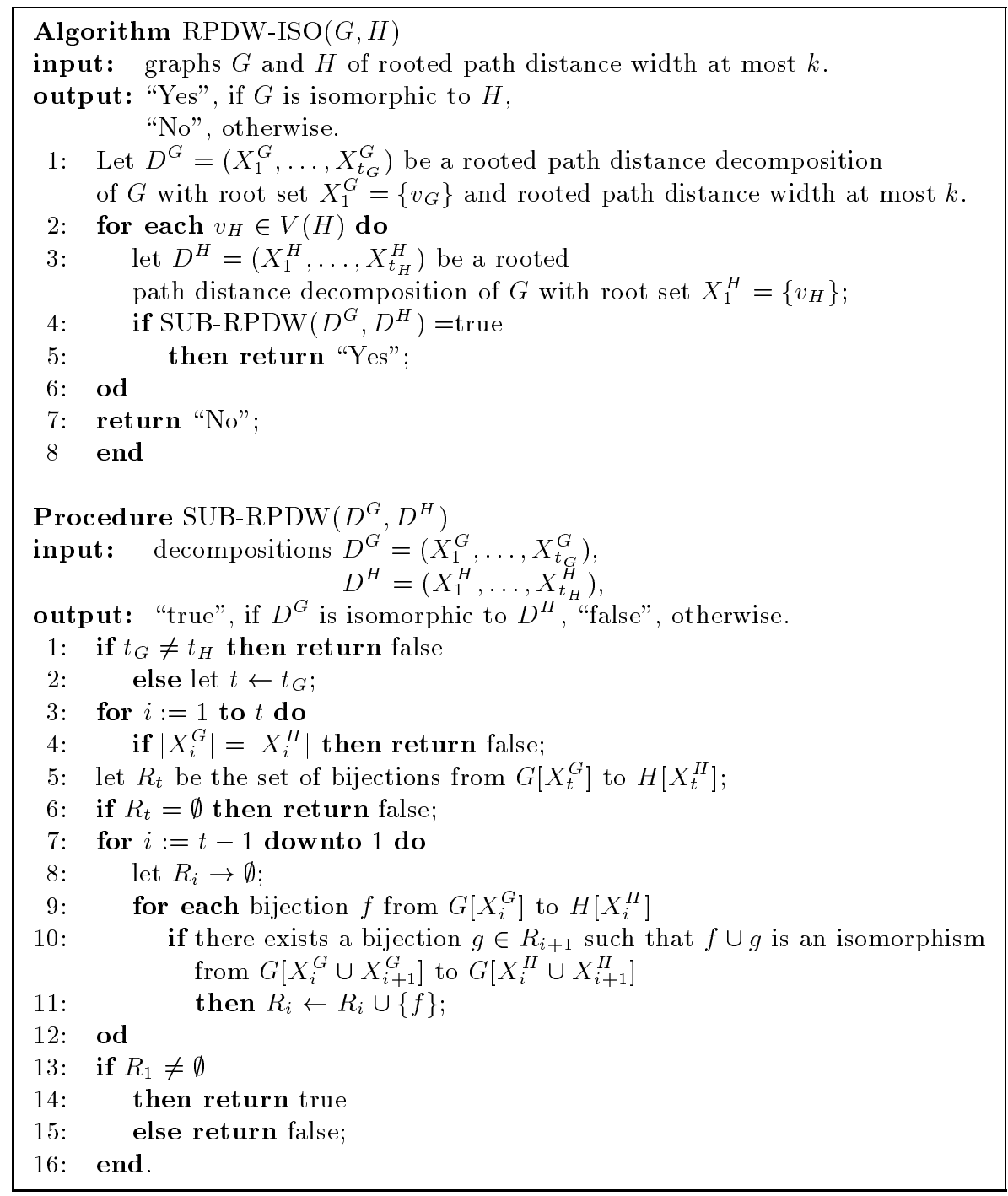

Definition Let $D^{G}=\left(X_{1}^{G}, \ldots, X_{t_{G}}^{G}\right)$ and $D^{H}=\left(X_{1}^{H}, \ldots, X_{t_{H}}^{H}\right)$ be two path distance decompositions of graphs $G$ and $H$ respectively. We call $D^{G}$ and $D^{H}$ isomorphic if there exists an isomorphism $f: V(G) \rightarrow V(H)$ from $G$ to $H$ such that for all $i, 1 \leq i \leq t$, $\forall v \in X_{i}^{G} f(v) \in X_{i}^{H}$. 
Theorem 3.1 The algorithm $\operatorname{RPDW}-\operatorname{ISO}(G, H)$ above, checks whether two input graphs $G$ and $H$ of rooted path distance width at most $k$ are isomorphic. The algorithm runs in $O\left(k !^{2} k^{2} n^{2}\right)$ time, where $n=|V(G)|$.

Proof. For input graphs $G$ and $H$, the algorithm works as follows. There are two phases. In the first phase (step 1 of $\operatorname{RPDW-ISO}(G, H)$ ), a rooted path distance decomposition of minimum width is computed for $G$. By Corollary 2.2 this phase costs $O\left(k n^{2}\right)$ time (as $|E(G)|=O(k|V(G)|)$ ).

In the second phase of the algorithm, we compute for each $v_{H} \in V(H)$ the unique rooted path distance decomposition $D^{H}=\left(X_{1}^{H}, \ldots, X_{t_{H}}^{H}\right)$ of $H$ where $X_{1}^{H}=\left\{v_{H}\right\}$ (step 3 of RPDW-ISO $(G, H)$ ). The main part of the algorithm is step 4 where procedure SUB-RPDW $\left(D^{G}, D^{H}\right)$ computes whether decompositions $D^{G}$ and $D^{H}$ are isomorphic. If $D^{G}$ and $D^{H}$ are isomorphic, then we may conclude that $G$ and $H$ are isomorphic. On the other hand, if there is no rooted path distance decomposition of $H$ which is isomorphic to $D^{G}$, then $G$ and $H$ cannot be isomorphic: if $f$ is an isomorphism from $G$ to $H$, then the rooted path decomposition of $H$ with $X_{1}^{H}=\left\{f\left(v_{G}\right)\right\}$ must be isomorphic to the rooted path decomposition of $G$ with $X_{1}^{G}=\left\{v_{G}\right\}$. So the given algorithm correctly computes whether $G$ and $H$ are isomorphic.

What now remains is to show that, given two path distance decompositions $D^{G}$ and $D^{H}$, SUB-RPDW $\left(D^{G}, D^{H}\right)$ indeed checks whether they are isomorphic. During steps 1-4 SUB-RPDW $\left(D^{G}, D^{H}\right)$ checks if $t_{G}=t_{H}$ and whether for $i=1, \ldots, t_{G}\left|X_{i}^{G}\right|=$ $\left|X_{i}^{H}\right|$. If not, then clearly $D^{G}$ and $D^{H}$ cannot be isomorphic. In the sequel (steps 512), for each $i, 1 \leq i \leq t=t_{G}$, $\operatorname{SUB}-\operatorname{RPDW}\left(D^{G}, D^{H}\right)$ computes the set $R_{i}$, which contains all isomorphisms from $G\left[X_{i}^{G}\right]$ to $H\left[X_{i}^{H}\right]$ that are extendible, i.e. $R_{i}$ contains all isomorphisms $f$ from $G\left[X_{i}^{G}\right]$ to $H\left[X_{i}^{H}\right]$ for which there is an isomorphism $g$ from $G\left[X_{i}^{G} \cup \ldots \cup X_{t}^{G}\right]$ to $H\left[X_{i}^{H} \cup \ldots, \cup X_{t}^{H}\right]$, such that for all $x \in X_{i}^{G}, f(x)=g(x)$ and for all $j, i \leq j \leq t$, for all $x \in X_{j}^{G}, g(x) \in X_{j}^{H}$. Now, it is clear that $R_{1}$ is not empty iff $D^{G}$ and $D^{H}$ are isomorphic. The set $R_{1}$ is computed in a bottom-up way, by first computing $R_{t}$, and then, for each $i, 1 \leq i \leq t-1$, computing $R_{i}$ from $R_{i+1}$. The set $R_{t}$ is easily computed by checking for each bijection $f: X_{t}^{G} \rightarrow X_{t}^{H}$, if it is an isomorphism. If so, SUB-RPDW $\left(D^{G}, D^{H}\right)$ puts $f$ in $R_{t}$ (step 5). This takes constant time, since there are at most $k$ ! such bijections.

For each $i, 1 \leq i<t$, SUB-RPDW $\left(D^{G}, D^{H}\right)$ computes $R_{i}$ as follows: for each bijection $f: X_{i}^{G} \rightarrow X_{i}^{H}$, checks if there is a $g \in R_{i+1}$, such that $f \cup g: X_{i}^{G} \cup X_{i+1}^{G} \rightarrow$ $X_{i}^{G} \cup X_{i+1}^{H}$ is an isomorphism from $G\left[X_{i}^{G} \cup X_{i+1}^{G}\right]$ to $H\left[X_{i}^{H} \cup X_{i+1}^{H}\right]$. If so, then put $f$ in $R_{i}$ (step 11). This can again be done in constant time. As there are no edges in $X_{i}^{G}$ $\left(X_{i}^{H}\right)$ adjacent with vertices in $X_{i+\sigma}^{G}\left(X_{i+\sigma}^{H}\right)$ for $\sigma=2, \ldots, t-i$, it is now easy to see that $R_{i}$ is the set of all the extendible isomorphisms from $X_{i}^{G}$ to $X_{i}^{H}$.

The running time of $\operatorname{RPWD} \operatorname{ISO}(G, H)$ is $O\left(k !^{2} k^{2} n^{2}\right)$ : computing the rooted path distance decomposition of $H$ for each possible root set take $O(k n)$ time. Furthermore, checking if two decompositions are isomorphic can be done in $O\left(k !^{2} k^{2} n\right)$ time: computing $R_{i}$ for some $i$ takes a constant time of $O\left(k !^{2} k^{2}\right)$, and there are at most $O(n)$ nodes.

Notice that it is not necessary that the input path decompositions of SUB-RPDW are rooted. Using this fact we can modify algorithm $\operatorname{RPDW-ISO}(G, H)$ in order to check isomorphism of graphs with small path distance width. 


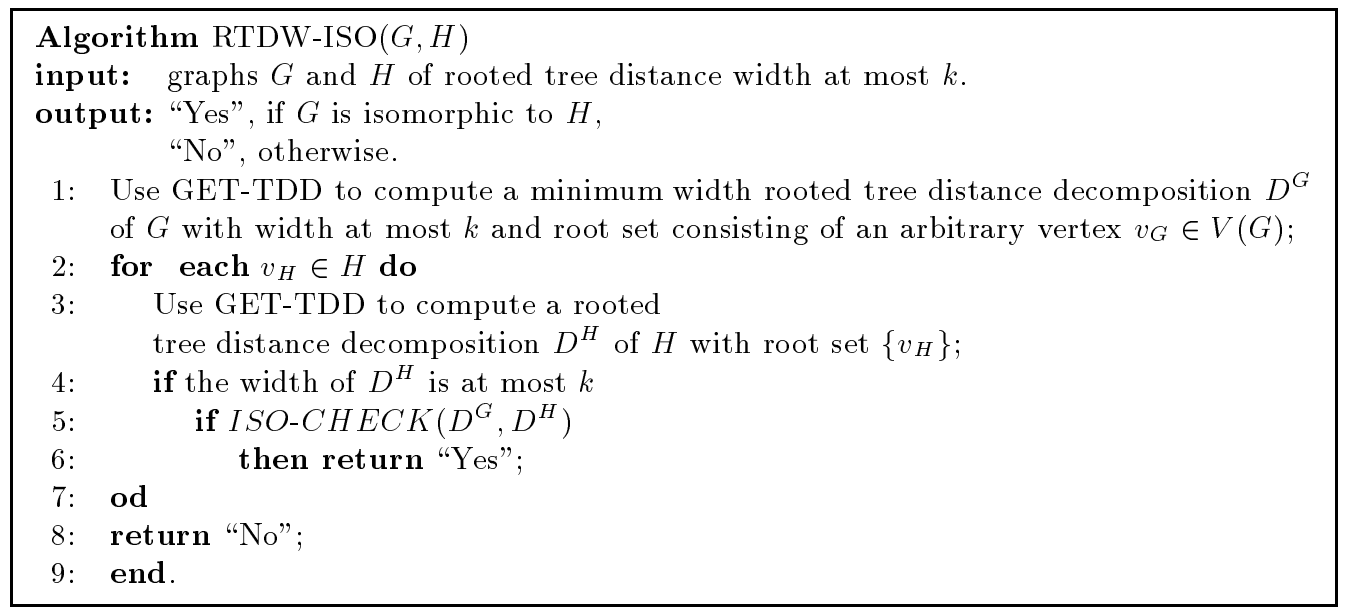

Figure 4: Algorithm RTDW-ISO.

Corollary 3.2 There exist an algorithm that checks whether two graphs $G$ and $H$ of path distance width at most $k$ are isomorphic in $O\left(k !^{2} k^{2} n^{k+1}\right)$ time $(n=|V(G)|)$.

Proof. We first compute an optimal path distance decomposition $D^{G}$ (this requires $O\left(n^{k}\right)$ time), and then we check, using $\operatorname{SUB}-\operatorname{RPDW}\left(D^{G}, D^{H}\right)$, if there exists some root set $X_{1}^{H} \subseteq V(H)\left(\left|X_{1}^{H}\right| \leq k\right)$ for which the corresponding path distance decomposition $D^{H}$ is isomorphic to $D^{G}$ (this requires $O\left(k !^{2} k^{2} n^{k+1}\right)$ time).

We now present an algorithm that computes whether two input graphs which have rooted tree distance width at most $k$ for some fixed $k$ are isomorphic. The running time of the algorithm is $O\left(n^{3}\right)$. The algorithm can be found in Figures 4, 5 and 6 .

Definition Let $D^{G}=\left(\left\{X_{i}^{G} \mid i \in I^{G}\right\}, T^{G}=\left(I^{G}, F^{G}\right), r_{G}\right)$ and $D^{H}=\left(\left\{X_{i}^{H} \mid i \in\right.\right.$ $\left.\left.I^{H}\right\}, T^{H}=\left(I^{H}, F^{H}\right), r_{H}\right)$ be two rooted tree distance decompositions of the graphs $G$ and $H$ respectively. We call $D^{G}$ and $D^{H}$ isomorphic if there exists an isomorphism $f: V(G) \rightarrow V(H)$ from $G$ to $H$ and an isomorphism $g: I^{G} \rightarrow I^{H}$ from $T^{G}$ to $T^{H}$ such that $g\left(r^{G}\right)=r^{H}$ and for each $i, i \in I^{G}$, and each $x \in I_{i}^{G}, f(x) \in I_{g(i)}^{H}$.

Theorem 3.3 Algorithm RTDW-ISO $(G, H)$ checks whether two input graphs $G$ and $H$ of rooted tree distance width at most $k$ are isomorphic. The algorithm runs in $O\left(k !^{2} k^{2} n^{3}\right)$ time, where $n=|V(G)|$.

Proof. The basic structure of the algorithm is the same as for graphs of bounded rooted path distance width: there are again two phases. In the first phase (step 1 of RTDW$\operatorname{ISO}(G, H)$ ), a rooted tree distance decomposition of minimum width is computed for $G$. This is done in the following way. For each vertex $v \in V$, GET-TDD is used to compute the unique minimal rooted tree distance decomposition of $G$ with root set $\{v\}$. Then, the decomposition $D^{G}$ of smallest width, say $k$ is selected. Let $D^{G}=\left(\left\{X_{i}^{G} \mid i \in\right.\right.$ $\left.\left.I^{G}\right\}, T^{G}=\left(I^{G}, F^{G}\right), r^{G}\right)$ denote this decomposition. By Corollary 2.2, this phase needs $O\left(k n^{2}\right)$ time. 


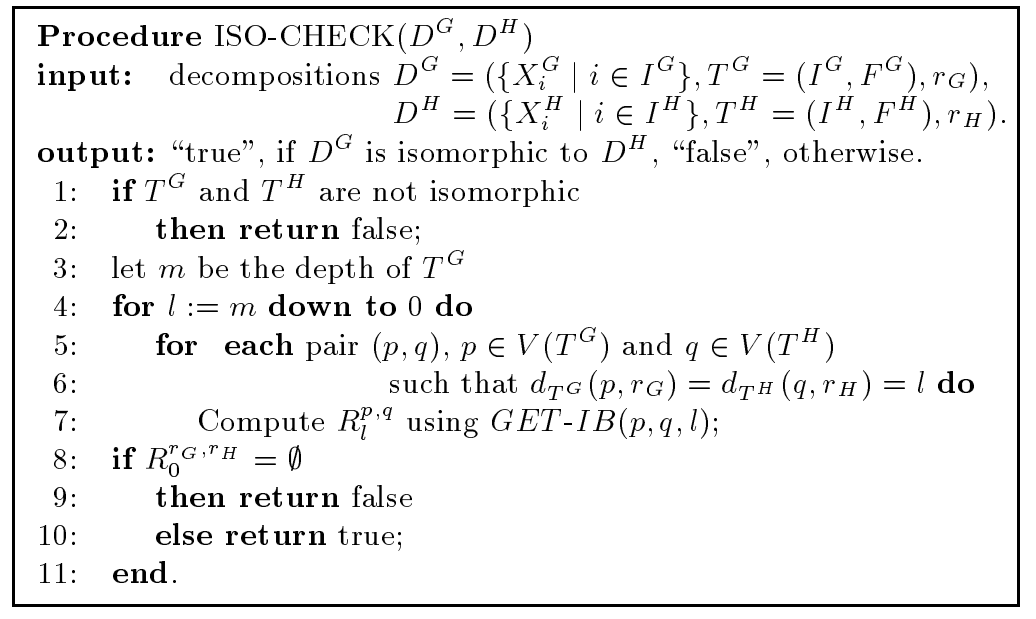

Figure 5: Procedure ISO-CHECK.

In the second phase of the algorithm (steps 2-11), RTDW-ISO $(G, H)$ computes, for each $w \in V(H)$, the unique minimal rooted tree distance decomposition $D^{H}$ of $H$ with root set $\{w\}$. Let $D^{H}=\left(\left\{X_{i}^{H} \mid i \in I^{H}\right\}, T^{H}=\left(I^{H}, F^{H}\right), r^{H}\right)$ denote such a decomposition. If the width of $D^{H}$ equals $k$, then procedure $\operatorname{ISO-CHECK}\left(D^{G}, D^{H}\right)$ is used to test whether decompositions $D^{G}$ and $D^{H}$ are isomorphic. In the same way as for the rooted path distance decompositions, we may conclude that $G$ and $H$ are isomorphic if $D^{G}$ and $D^{H}$ are isomorphic. On the other hand, if there is no minimal rooted tree distance decomposition of $H$ which is isomorphic to $D^{G}$, then $G$ and $H$ cannot be isomorphic. So the given algorithm correctly computes whether $G$ and $H$ are isomorphic, assumed that procedure ISO-CHECK is correct.

What now remains is to see that procedure $\operatorname{ISO} \operatorname{CHECK}\left(D^{G}, D^{H}\right)$ indeed tests whether two rooted tree distance decompositions are isomorphic.

Suppose $D^{G}=\left(\left\{X_{i}^{G} \mid i \in I^{G}\right\}, T^{G}=\left(I^{G}, F^{G}\right), r^{G}\right)$ and $D^{H}=\left(\left\{X_{i}^{H} \mid i \in\right.\right.$ $\left.\left.I^{H}\right\}, T^{H}=\left(I^{H}, F^{H}\right), r^{H}\right)$ are minimal rooted tree distance decompositions of graphs $G$ and $H$, respectively. Note that $D^{G}$ and $D^{H}$ cannot be isomorphic if $T^{G}$ and $T^{H}$ are not isomorphic. Therefore, ISO-CHECK $\left(D^{G}, D^{H}\right)$ first test whether $T^{G}$ and $T^{H}$ are isomorphic (step 1). This test uses the rooted tree isomorphism checking algorithm from [6] and requires $O(n)$ time. Now suppose $T^{G}$ and $T^{H}$ are isomorphic. The depth of a node in a rooted tree is the length of a path from this node to the root (so the root has depth zero). Let $m$ denote the maximum depth of a node in $T^{G}$ (and hence in $T^{H}$ ). The nodes of depth $l$ are called nodes on level $l$. Now, for each level $l, 0 \leq l \leq m$, and each pair of nodes $p, q$, with $p \in I^{G}$ and $q \in I^{H}$, both on level $l$, we compute the set $R_{l}^{p, q}$ of isomorphisms $f: X_{p}^{G} \rightarrow X_{q}^{H}$ from $G\left[X_{p}^{G}\right]$ to $H\left[X_{q}^{H}\right]$ which are extendible. The definition of an extendible isomorphism for tree distance decompositions is a generalisation of the one used in the proof of Theorem 3.1 and is the following:

Definition Let $G_{p}=G\left[V\left(D^{G}, p\right)\right]$ and $H_{q}=H\left[V\left(D^{H}, q\right)\right]$. We say an isomorphism $f$ from $G\left[X_{p}^{G}\right]$ to $H\left[X_{q}^{H}\right]$ is extendible if there exists an isomorphism $g^{\prime}: V\left(T_{p}^{G}\right) \rightarrow V\left(T_{q}^{H}\right)$ from $T_{p}^{G}$ to $T_{q}^{H}$ and an isomorphism $f^{\prime}: V\left(G_{p}\right) \rightarrow V\left(H_{q}\right)$ from $G_{p}$ to $H_{q}$ such that for 


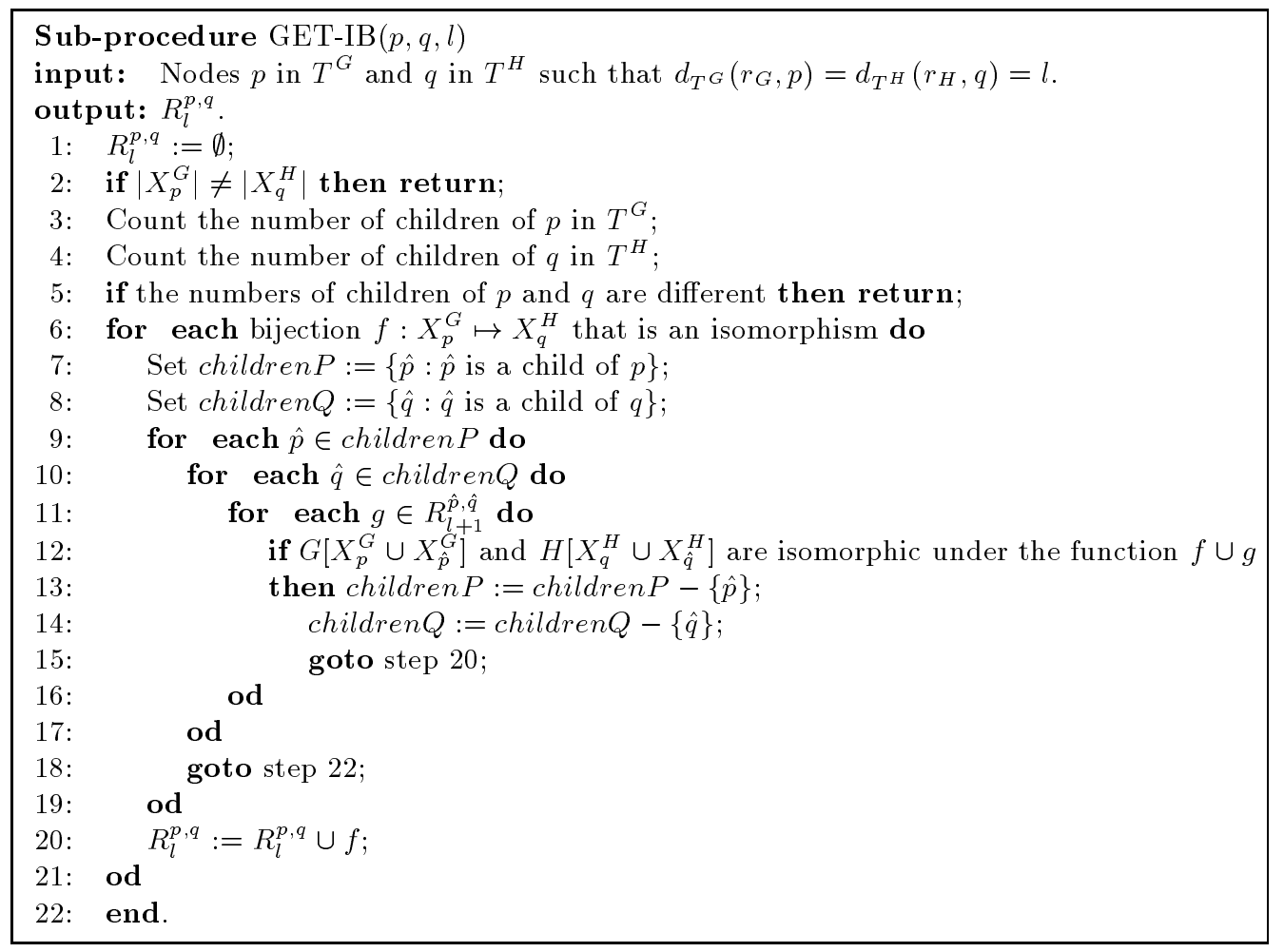

Figure 6: Sub-procedure GET-IB.

each $a \in V\left(T_{p}^{G}\right)$ and each $v \in X_{a}^{G}, f^{\prime}(v) \in X_{g^{\prime}(a)}^{H}$, and furthermore, for each $v \in X_{p}^{G}$, $f(v)=f^{\prime}(v)$ (see Figure 7).

From the definition above it is clear that $R_{0}^{r^{G}, r^{H}}$ is not empty iff $D^{G}$ and $D^{H}$ are isomorphic. The set $R_{0}^{r^{G}, r^{H}}$ is computed in a bottom-up way, by first computing $R_{m}^{p, q}$ for all nodes $p \in I^{G}$ and $q \in I^{H}$ on level $m$, and then, for each $l, 0 \leq l<m$, computing $R_{l}^{p, q}$ for all nodes $p \in I^{G}$ and $q \in I^{H}$ on level $l$, by using the values of $R_{l+1}^{\hat{p}, \hat{q}}$ for all children $\hat{p}$ of $p$ and $\hat{q}$ of $q$.

The sets $R_{m}^{p, q}$ ( $p$ and $q$ are nodes of level $m$ in $T^{G}$ and $T^{H}$ respectively) are computed during the first execution of loop $4-7$ of $\operatorname{ISO}-\operatorname{CHECK}\left(D^{G}, D^{H}\right)$ : if $\left|X_{p}^{G}\right| \neq\left|X_{q}^{H}\right|$, then $R_{m}^{p, q}=\emptyset$ (step 2 of $\left.\operatorname{GET}-\operatorname{IB}(p, q, l)\right)$. Otherwise, for each bijection $f: X_{p}^{G} \rightarrow X_{q}^{H}$, GET$\operatorname{IB}(p, q, l)$ checks if it is an isomorphism (step 6), and if so, puts $f$ in $R_{m}^{p, q}$ (step 21). This takes constant time for each $p$ and $q$.

For each $l, 0 \leq l<m$, and each $p \in I^{G}$ and $q \in I^{H}$ on level $l$ of $T^{G}$ and $T^{H}$ respectively, $\operatorname{GET}-\operatorname{IB}(p, q, l)$ proceeds computing $R_{l}^{p, q}$ as follows. First, it checks if $\left|X_{p}^{G}\right|=\left|X_{q}^{H}\right|$ and the number of children of $p$ equals the number of children of $q$. If not, then $R_{l}^{p, q}=\emptyset$. Otherwise, for each bijection $f: X_{p}^{G} \rightarrow X_{q}^{H}$ from $G\left[X_{p}^{G}\right]$ to $H\left[X_{q}^{H}\right]$ that is an isomorphism, $\operatorname{GET}-\operatorname{IB}(p, q, l)$ tries to make a matching between the children of $p$ and the children of $q$, i.e., it tries to match each child $\hat{p}$ of $p$ to a child $\hat{q}$ of $q$, in such a way that there exists a $g \in R_{l+1}^{\hat{p}, \hat{q}}$ for which $f \cup g: X_{p}^{G} \cup X_{\hat{p}}^{G} \rightarrow X_{q}^{H} \cup X_{\hat{q}}^{H}$ is an 


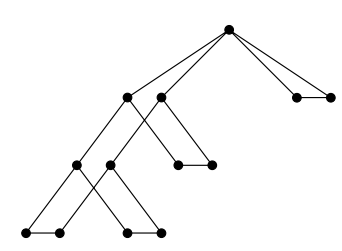

Graph $G, H$

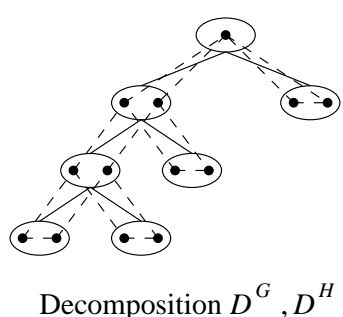

Decomposition $D^{G}, D^{H}$

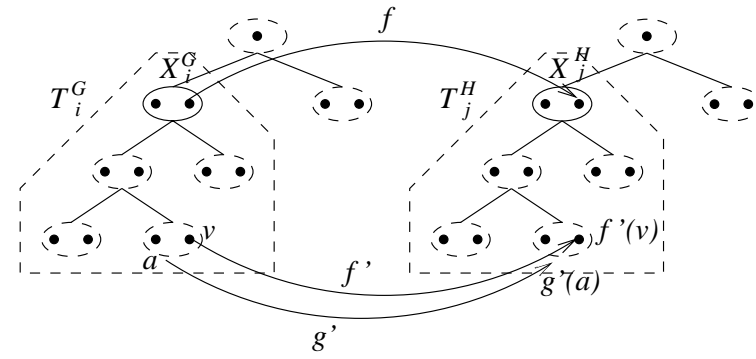

The functions $f, f$ ' and $g$ '

Figure 7: An example of extendibility of isomorphism from $G\left[X_{i}^{G}\right]$ to $H\left[X_{j}^{H}\right]$.

isomorphism from $G\left[X_{p}^{G} \cup X_{\hat{p}}^{G}\right]$ to $H\left[X_{q}^{H} \cup X_{\hat{q}}^{H}\right]$ (steps 9-20). As there are no vertices in $X_{p}^{G}\left(X_{q}^{H}\right)$ adjacent with vertices belonging to some $X_{\sigma}^{G}\left(X_{\sigma^{\prime}}^{H}\right)$ where $\sigma\left(\sigma^{\prime}\right)$ is an ancestor of $p(q)$ in $T^{G}\left(T^{H}\right)$ that is not a child of $p(q)$, it is easy to see that there exists such a matching iff $f$ is extendible (see Figure 8 ).

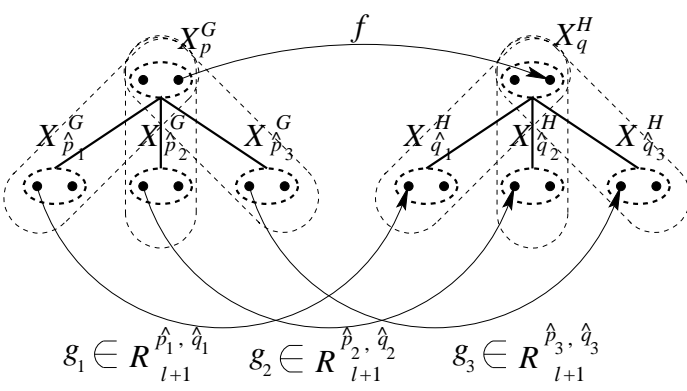

Figure 8: An example of a matching between children of $X_{i}^{G}$ and $X_{j}^{H}$.

Towards computing a matching as described above, $\operatorname{GET}-\operatorname{IB}(p, q, l)$ tries to match the children of $p$ one by one to a child of $q$ as follows: Take a child $\hat{p}$ of $p$ which has not yet been matched (step 9). For each unmatched child $\hat{q}$ of $q$, try to match $\hat{p}$ to $\hat{q}$ (steps 10-18). As soon as a $\hat{q}$ is found that can be matched to $\hat{p}$, then match $\hat{p}$ to $\hat{q}$, and go on with the next child of $p$. If there is no $\hat{q}$ which can be matched to $\hat{p}$, then there cannot be a matching between the children of $p$ and of $q$, and hence $f$ is not extendible. On the other side, it is clear that if $f$ is extendible, such a matching exists. Now, if it is possible to match each child of $p$ to a child of $q$, then $\operatorname{GET}-\operatorname{IB}(p, q, l)$ adds $f$ to $R_{l}^{p, q}$ (step 21). (We can actually use this 'greedy matching algorithm', and need not use the standard 'maximum flow' matching algorithm, because of transitivity of isomorphism.)

We can easily observe that during the $l$ th execution of the loop defined by steps $4-7$ of ISO-CHECK $\left(D^{G}, D^{H}\right)$, the number of the execution times of the loop defined by steps 11-17 of $\operatorname{GET}-\operatorname{IB}(p, q, l)$ is quadratic in the number of edges in $T^{G}$ (or $\left.T^{H}\right)$ that have one endpoint in level $l$ and one in level $l+1$. As the number of edges in $T^{G}$ is 
$O(n)$ we can easily conclude that the overall complexity of $\operatorname{ISO} \operatorname{CHECK}\left(D^{G}, D^{H}\right)$ is $O\left(k !^{2} k^{2} n^{2}\right)$.

The running time of the second phase of algorithm $\operatorname{RTWD-ISO}(G, H)$ is $O\left(k !^{2} k^{2} n^{3}\right)$ : computing the minimal rooted tree distance decomposition of $H$ for a root set $\{w\}$ takes $O\left(n^{2}\right)$ time. Moreover, as ISO-CHECK $\left(D^{G}, D^{H}\right)$ needs $O\left(k !^{2} k^{2} n^{2}\right)$ time and the number of different root sets for $H$ is $n$, the running time of $O\left(k ! !^{2} k^{2} n^{3}\right)$ now follows.

Notice that it is not necessary that the inputs of $\operatorname{ISO}-\operatorname{CHECK}\left(D^{G}, D^{H}\right)$ are rooted. Using this fact we can modify algorithm $\operatorname{RTDW-ISO}(G, H)$ in order to check isomorphism of graphs with small tree distance width.

Corollary 3.4 There exist an algorithm that checks whether two graphs $G$ and $H$ of path distance width at most $k$ are isomorphic in $O\left(k !^{2} k^{2} n^{k+2}\right)$ time $(n=|V(G)|)$.

Proof. We first compute an optimal path distance decomposition $D^{G}$ (this requires $O\left(n^{k}\right)$ time), and then we check, using ISO-CHECK $\left(D^{G}, D^{H}\right)$, if there exists some root set $X_{r_{H}}^{H} \subseteq V(H)\left(\left|X_{r_{H}}^{H}\right| \leq k\right)$ for which the corresponding tree distance decomposition $D^{H}$ is isomorphic to $D^{G}$. This requires $O\left(n^{k}\right)$ calls of $\operatorname{ISO} \operatorname{CHECK}\left(D^{G}, D^{H}\right)$ and thus $O\left(k !^{2} k^{2} n^{k+2}\right)$ time.

\section{Relationships Among Classes of Bounded Width}

In this section, first we give the definitions of some known graph parameters in order to investigate their relations with (rooted) path distance width and (rooted) tree distance width.

- A strong tree decomposition of a graph $G=(V, E)$ is a pair $\left(\left\{X_{i} \mid i \in I\right\}, T=\right.$ $(I, F)$ ), where $\left\{X_{i} \mid i \in I\right\}$ is a collection of subsets of $V$ and $T$ is a tree, such that

$-\bigcup_{i \in I} X_{i}=V$ and for all $i \neq j, X_{i} \cap X_{j}=\emptyset$,

- for each edge $\{v, w\} \in E$, there are $i, j \in I$ with $v \in X_{i}$ and $w \in X_{j}$, such that either $i=j$ or $\{i, j\} \in F$.

The width of a strong tree decomposition $\left(\left\{X_{i} \mid i \in I\right\}, T=(I, F)\right)$ equals $\max _{i \in I}\left(\left|X_{i}\right|\right)$. The strong treewidth of a graph $G$ is the minimum width over all possible strong tree decompositions of $G$. The corresponding graph parameter is denoted by $\mathcal{S T} \mathcal{W}$.

- A connected strong tree decomposition of a graph $G=(V, E)$ is a strong tree decomposition $\left(\left\{X_{i} \mid i \in I\right\}, T=(I, F)\right)$ of $G$ such that for each $i \in I, G\left[X_{i}\right]$ is connected. The connected strong treewidth of $G$ is the minimum width over all connected strong tree decompositions of $G$. The corresponding graph parameter is denoted by $\mathcal{C S T \mathcal { W }}$.

- A path decomposition of a graph $G$ is a tree decomposition $\left(\left\{X_{i} \mid i \in I\right\}, T=\right.$ $(I, F)$ ) in which $T$ is a path (i.e. two nodes in $T$ have degree one, and all others have degree two). The pathwidth of a graph $G$ is the minimum width over all path decompositions of $G$. The corresponding graph parameter is denoted by $\mathcal{P W}$. 
In the same way, we can define the notions of strong pathwidth, and connected strong pathwidth. We denote the corresponding graph parameters by $\mathcal{S P W}$ and $\mathcal{C S P W}$, respectively.

- The cutwidth of a layout $f$ of a graph $G$ is defined as

$$
\max _{1 \leq i<|V(G)|}|\{\{u, v\} \in E(G): f(u) \leq i<f(v)\}| .
$$

The cutwidth of a graph $G$ is the minimum cutwidth over all layouts of $G$. The corresponding graph parameter is denoted by $\mathcal{C W}$.

- For a given graph $G$, a subdivision is the operation which adds a new vertex $u$ to $G$ and replaces an edge $e=\{v, w\} \in E(G)$ by two edges $\{v, u\}$ and $\{u, w\}$ (i.e. it splits an edge of $G$ into two edges). A refinement of a graph $G$ is a graph $G^{\prime}$ which is obtained from $G$ by a number of subsequent subdivisions.

- The topological bandwidth of a graph $G$ is the minimum bandwidth over all refinements of $G$. The corresponding graph parameter is denoted by $\mathcal{T} \mathcal{B W}$.

- By $\mathcal{D}$ we denote the graph parameter which maps each graph to the maximum degree of any vertex in the graph.

Let $f$ and $f^{\prime}$ be two graph parameters. We say that $f^{\prime}$ covers $f$, denoted by $f \preceq f^{\prime}$, if there is a function $g: \mathbf{N} \rightarrow \mathbf{N}$, such that for each graph $G$ and each integer $k$, if $f(G) \leq k$ then $f^{\prime}(G) \leq g(k)$ (we also say that $f$ is covered by $f^{\prime}$ ). For instance, if we take $f=\mathcal{B} \mathcal{W}$ and $f^{\prime}=\mathcal{C W}$, then $f \preceq f^{\prime}$ : for each graph $G, \mathcal{C W}(G) \leq \mathcal{B W}(G)(\mathcal{B W}(G)-1) / 2$. Hence if we take $g(k)=k(k-1) / 2$, then for each graph $G$ and each integer $k$, if $\mathcal{B W}(G) \leq k$ then $\mathcal{C W}(G) \leq g(k)$.

If a graph parameter $f$ is not covered by a parameter $f^{\prime}$, we denote this by $f \npreceq f^{\prime}$. If $f \preceq f^{\prime}$ but $f^{\prime} \npreceq f$, then we say that $f^{\prime}$ strictly covers $f$, denoted by $f \prec f^{\prime}$. If $f \preceq f^{\prime}$ and $f^{\prime} \preceq f$, then we say $f \approx f^{\prime}$. If $f \npreceq f^{\prime}$ and $f^{\prime} \npreceq f$, then we say that $f$ and $f^{\prime}$ are not related, and we denote this by $f \not f^{\prime}$ (note that saying that $f \not \approx f^{\prime}$ is not equivalent to saying that $f \approx f^{\prime}$ does not hold). It is easy to see that $\prec, \preceq$ and $\approx$ are transitive relations.

The notion of covering is interesting in the following sense. Suppose we have a graph problem $P$ (for example the isomorphism problem), and we have two graph parameters $f$ and $f^{\prime}$, such that $f \preceq f^{\prime}$. If problem $P$ is fixed parameter tractable for parameter $f^{\prime}$, then we can conclude immediately that $P$ is fixed parameter tractable for $f$. On the other hand, if we can show that problem $P$ is fixed parameter tractable for parameter $f$, then this might help to get more insight in whether $P$ is fixed parameter tractable for parameter $f^{\prime}$.

We now give a number of relations for the graph parameters that are defined in Section 2 and this section.

Theorem 4.1 The following relations hold (see also Figure 9).
(1) $\mathcal{T W} \not \mathcal{D}$
(2) $\mathcal{C W} \prec \mathcal{T W}$
(3) $\mathcal{C W} \prec \mathcal{D}$
(4) $\mathcal{C W} \approx \mathcal{T} \mathcal{B W}$
(5) $\mathcal{B W} \prec \mathcal{C W}$
(6) $\mathcal{S P W} \approx \mathcal{B W}$
(7) $\mathcal{R} \mathcal{T} \mathcal{D W} \npreceq \mathcal{B W}$
(8) $\mathcal{R} \mathcal{P} \mathcal{D W} \prec \mathcal{P} \mathcal{D W}$
(9) $\mathcal{P D W} \prec \mathcal{B W}$
(10) $\mathcal{C S P W} \prec \mathcal{R P D W}$
(11) $\mathcal{C S T W} \not \approx \mathcal{T} \mathcal{D W}$
(12) $\mathcal{R} \mathcal{T} \mathcal{D W} \not \approx \mathcal{C S T W}$ 


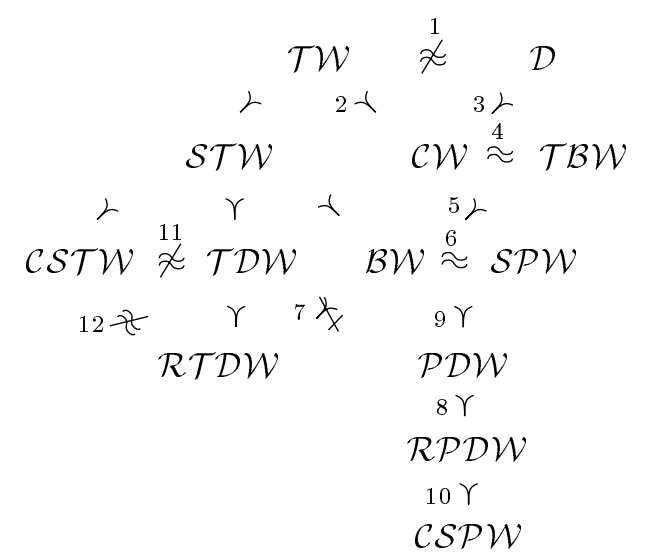

Figure 9: Relations between graph parameters.

Proof. (1) In order to see that $\mathcal{T W} \npreceq \mathcal{D}$ it is sufficient to observe that trees have treewidth 1 and arbitrary large maximum degree. For $\mathcal{D} \npreceq \mathcal{T} \mathcal{W}$, we may notice that grids have maximum degree $\leq 4$ and arbitrary large treewidth (see [13]).

(2) $\mathcal{C W} \preceq \mathcal{T W}$ follows immediately from the fact that for any graph $G, \mathcal{T W}(G) \leq$ $\mathcal{C W}(G)$ (see [1]). Also, it is known that for any complete binary tree $B_{k}$ with depth $k \geq 2 \quad \mathcal{C} \mathcal{W}\left(B_{k}\right)=\lceil(k-1) / 2\rceil+1$ (see [4]). Hence $\mathcal{T} \mathcal{W} \npreceq \mathcal{C W}$.

(3) It is easy to see that the $\mathcal{C W}(G) \geq \mathcal{D}(G) / 2$ for any graph $G$. Thus, $\mathcal{C W} \preceq \mathcal{D}$. Moreover, as we mention in the proof of $(2)$, the complete binary trees $B_{k}$ with depth $k>3$, have cutwidth equal to $\lceil(k-1) / 2\rceil+1$. As $\Delta\left(B_{k}\right)=3$ we have that $\mathcal{D} \npreceq \mathcal{C W}$.

(4) It is known that $\mathcal{T} \mathcal{B W}(G) \leq \mathcal{C W}(G)$ for any graph $G$ (see [4]), and that there exists a function $f$ such that for any graph $G, \mathcal{C W}(G) \leq f(\mathcal{T B W}(G)$ ) (see [5]). Hence, we have $\mathcal{C W} \preceq \mathcal{T} \mathcal{B W}$ and $\mathcal{T} \mathcal{B W} \preceq \mathcal{C W}$.

(5) In [1], it is shown that for any graph $G, \mathcal{B W}(G)(\mathcal{B W}(G)+1) / 2 \geq \mathcal{C W}(G)$. Therefore, $\mathcal{B W} \preceq \mathcal{C W}$.

Consider the class $L$ of graphs shown in Figure 10. It is clear that these graphs have bounded cutwidth but arbitrary large bandwidth (use the well known formula $\frac{|V(G)|-1}{\operatorname{diam}(G)} \leq \mathrm{BW}(G)$, where $\operatorname{diam}(G)$ is the diameter of $G$, see $\left.[5]\right)$. Thus $\mathcal{C W} \npreceq \mathcal{B W}$.

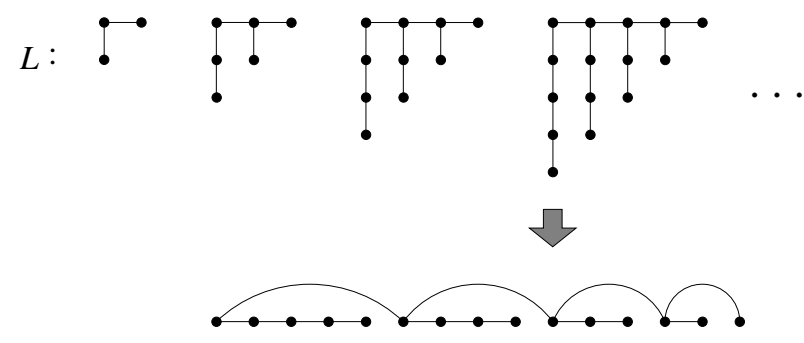

Figure 10: A counterexample for $\mathcal{C W} \npreceq \mathcal{B} \mathcal{W}$ 
(6) Let $G$ be a graph which has a strong path decomposition $\left(X_{1}, X_{2}, \cdots, X_{t}\right)$ with width at most $k$. We will prove that the bandwidth of $G$ is bounded by $g(k)=2 k-1$. Consider a linear layout 1 such that if $u \in X_{i}, v \in X_{j}$, and $i<j$ then $f(u)<f(v)$. Let $\{u, v\}$ be an edge in $G$, and let $i$ and $j$ be the subscripts such that $u \in X_{i}$ and $v \in X_{j}$. Since $\left|X_{h}\right| \leq k$ for each $h, 1 \leq h \leq t$ and $|i-j| \leq 1,|l(u)-l(v)| \leq 2 k-1$. Hence $G$ has bandwidth $\leq 2 k-1$. Thus, we have that $\mathcal{S P W} \preceq \mathcal{B W}$.

Let $G$ be a graph with bandwidth $\leq k$. We will prove that $\mathcal{S P W}(G) \leq k$. There exists a linear layout $f$ such that for all $\{u, v\} \in E(G),|f(u)-f(v)| \leq k$. For each $i$, $1 \leq i \leq\lceil n / k\rceil$, let $X_{i}=\{u \mid u \in V(G),(i-1) k+1 \leq f(u) \leq i k\}(n=|V(G)|)$. Clearly, $\left(X_{1}, X_{2}, \cdots, X_{\lceil n / k\rceil}\right)$ is a strong path decomposition with strong pathwidth $k$. Thus we have that $\mathcal{B W} \preceq \mathcal{S P W}$.

(7) It is easy to see that $\mathcal{R T} \mathcal{D W} \npreceq \mathcal{B W}$ by considering the class of complete binary trees. (It is well known that the bandwidth of a $k$-depth complete binary tree is $\left\lceil\left(2^{k}-1\right) / k\right\rceil$, see [15].)

(8) We straightforwardly obtain $\mathcal{R} \mathcal{P} \mathcal{D} \mathcal{W} \preceq \mathcal{P D} \mathcal{W}$ from the definitions.

Consider the class $L$ of graphs described in Figure 11. It is clear that any graph in $L$ has bounded path distance width and arbitrary large rooted path distance width. Thus $\mathcal{P D} \mathcal{W} \npreceq \mathcal{R} \mathcal{P} \mathcal{D} \mathcal{W}$.

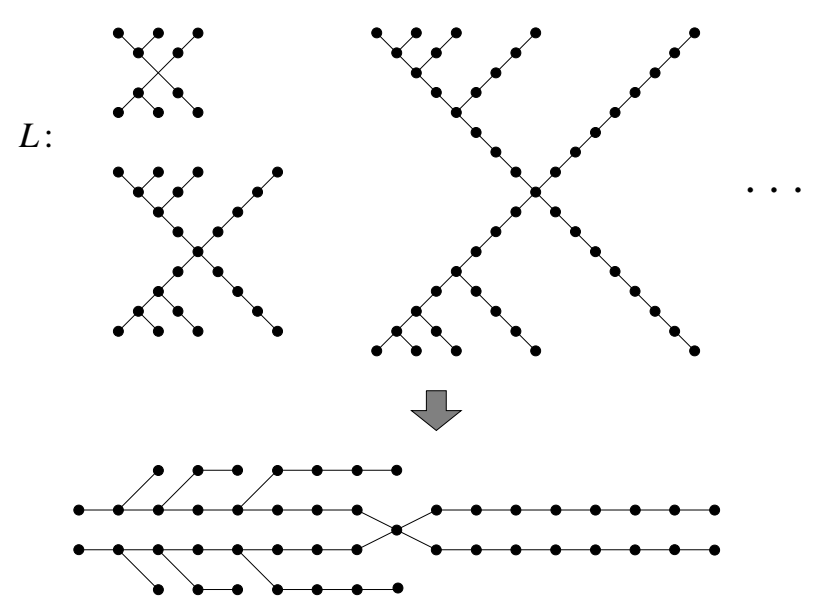

Figure 11: A counterexample for $\mathcal{P D} \mathcal{W} \npreceq \mathcal{R} \mathcal{P} \mathcal{D} \mathcal{W}$.

(9) As any path distance decomposition is also a strong path decomposition of the same width, we have that $\mathcal{P} \mathcal{D W} \preceq \mathcal{S P W}$ (and hence $\mathcal{P} \mathcal{D W} \preceq \mathcal{B W}$ ).

We prove that $\mathcal{S P W} \npreceq \mathcal{P D \mathcal { W }}$. We call the graph in Figure 12(a) a double ribbon with size $k$ (in Figure 12(a) $k=3$ ). We call the rightmost vertex and the leftmost vertex in a double ribbon endpoints. The middle vertex in a double ribbon is called the center. The strong pathwidth of $H_{k}$ is at most 3 for each $k$ (see Figure 12(b,c)). We show that for each $k$, the path distance width of $H_{k}$ is at least $k+1$. Suppose, on the contrary, that there exists a path distance decomposition of $H_{k}$ with root set $S$ and width at most $k$. Since the size of $S$ is at most $k$, there exists at least one double ribbon $R$ which does not have vertices in $S$. Let $a$ and $b$ be the endpoints of $R$, and let $c$ be the center of 
$R$. We set $d_{a}=d_{H_{k}}(S, a), d_{b}=d_{H_{k}}(S, b), d_{c}=d_{H_{k}}(S, c)$. Without loss of generality, we assume that $d_{a} \leq d_{b}$. Then we have $d_{c}=d_{a}+2^{k}+2^{k}=d_{a}+2^{k+1}$ and this means that there exist at least $k+2$ vertices with distance $d_{a}+2^{k+1}$ which is a contradiction (see Figure 12(a)). Hence, we have $\mathcal{S P W} \npreceq \mathcal{P D \mathcal { W }}$ (and thus $\mathcal{B W} \npreceq \mathcal{P D \mathcal { W }}$ ).

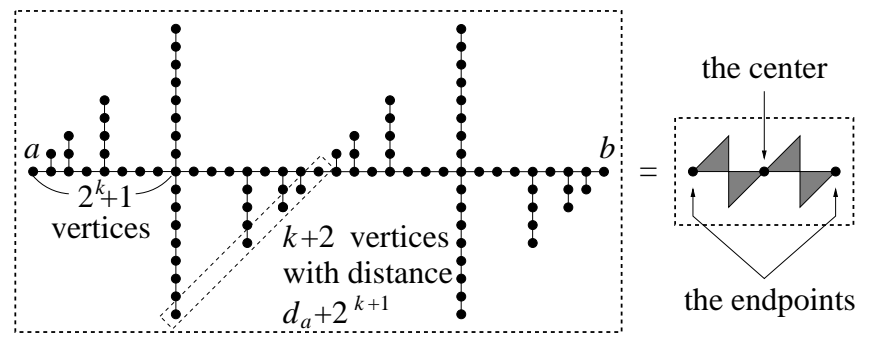

(a)

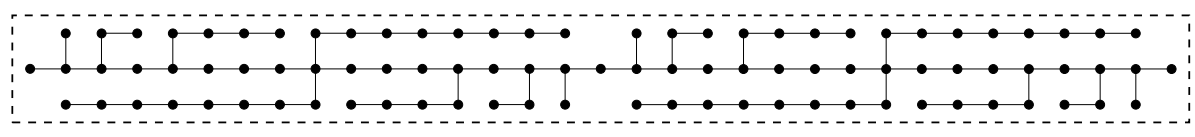

(b)

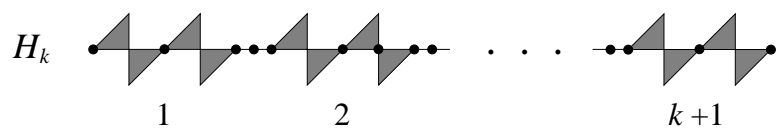

(c)

Figure 12: A counterexample for $S \mathcal{P W} \npreceq \mathcal{P D} \mathcal{W}$.

(10) Let $G$ be a graph with $\operatorname{CSPW}(G) \leq k$. We show that $\mathcal{R P D} \mathcal{W}(G) \leq k^{2}$. Let $\left(X_{1}, X_{2}, \ldots, X_{m}\right)$ be a connected strong path decomposition of $G$ of width at most $k$. We will construct a rooted path distance decomposition of $G$ of width at most $k^{2}$.

Let $r$ be an arbitrary vertex from $X_{1}$, let $\{r\}$ be the root set. Furthermore, let $L_{i}=\max _{v \in X_{i}} d_{G}(r, v)$, $S_{i}=\min _{v \in X_{i}} d_{G}(r, v)$, $\operatorname{rt}(i)=\max \left\{j \mid X_{j}\right.$ contains a vertex $v$ with $\left.d_{G}(r, v)=i\right\}$ and $\operatorname{lt}(i)=\min \left\{j \mid X_{j}\right.$ contains a vertex $v$ with $\left.d_{G}(r, v)=i\right\}$. For example, in Figure 13 $L_{2}=3, S_{2}=1, \operatorname{rt}(3)=4$, and $\operatorname{lt}(3)=2$.

Notice that (i) as $G\left[X_{i}\right]$ is connected, we have that for all $i, 1 \leq i \leq m, L_{i}-S_{i} \leq k-1$, (ii) for all $i, 1 \leq i \leq m-1, S_{i+1}-S_{i} \geq 1$ and (iii) for all $i, 1 \leq i \leq m-1$, for all $j$, $1 \leq j \leq m-i, S_{i}+j \leq S_{i+j}$.

We will now show that $\forall d, 1 \leq d \leq \max _{v \in V(G)} d_{G}(r, v), \operatorname{rt}(d)-\operatorname{lt}(d)<k$, or in other words, the number of sets $X_{i}$ which have a vertex with distance $d$ from the root is at most $k$. Suppose, on the contrary, that $\operatorname{lt}(d)+k \leq \operatorname{rt}(d)$ for some $d$. Since there exists a vertex with distance $d$ in $X_{\mathrm{lt}(d)}$ and because of (i), we have that

$$
d \leq L_{\mathrm{lt}(d)} \leq S_{\mathrm{lt}(d)}+k-1 .
$$




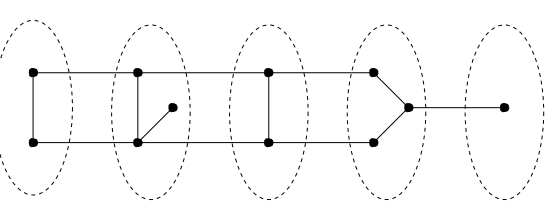

A connected strong path decomposition

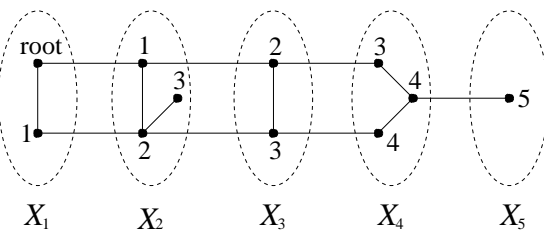

A labeled decomposition

Figure 13: An example of labelled decomposition.

Also, using (ii), (iii), the assumption above and the fact that there exists a vertex in $X_{\mathrm{rt}(d)}$ with distance $d$ from the root, we have that

$$
S_{\mathrm{lt}(d)}+k \leq S_{\mathrm{lt}(d)+k} \leq S_{\mathrm{rt}(d)} \leq d .
$$

From (1) and (2) we have that $d \leq S_{\mathrm{lt}(d)}+k-1<S_{\mathrm{lt}(d)}+k \leq d$, which is a contradiction. Hence, for any integer $d$ the number of the vertices of the strong path decomposition that have a vertex with distance $d$ from the root is at most $k$. Therefore, the number of vertices having distance $d$ from the root is at most $k^{2}$ and thus, we can construct a rooted path distance decomposition of $G$ with width at most $k^{2}$.

It is easy to see (using the class of cycles as a counterexample) that $\mathcal{R} \mathcal{P D W} \npreceq$ $\mathcal{C S P W}$.

(11) Consider the class of graphs $G_{k}^{\prime} k \geq 1$ in Figure 14. Clearly, these graphs have connected strong treewidth equal to 2 . Now, towards proving $\mathcal{C S T W} \npreceq \mathcal{T} \mathcal{D W}$, we will show that for any $k$, the tree distance width of $G_{k}$ is greater than or equal to $k+1$. Suppose, on the contrary that there exists a tree distance decomposition of $G_{k}$ with root set $S$ and width at most $k$. Since the size of $S$ is at most $k$, there exists at least one copy, say $H$, of $G_{k}$ in $G_{k}^{\prime}$ such that $H$ does not have vertices in $S$. Let $b_{i}$ be the base (see Figure 14) of $H$, and $d_{b_{i}}$ be the distance between $S$ and $b_{i}$ in $G_{k}^{\prime}$. It is not hard to see that $H$ has exactly $k+1$ vertices which are of distance $d_{b_{i}}+1$ from $S$, a contradiction. Finally, using again the class of cycles as a counter example, we have $\mathcal{T} \mathcal{D W} \npreceq \mathcal{C S T \mathcal { W }}$.

(12) It is easy to see that $\mathcal{R T D W} \npreceq \mathcal{C S T \mathcal { W }}$ (the class of cycles is again a counterexample). Using again the class of graphs in Figure 14 as a counter example, we have $\mathcal{C S T W} \npreceq \mathcal{R} \mathcal{T} \mathcal{D W}$.

An immediate consequence of the above relations and Theorem 3.1 is that graph isomorphism is fixed parameter tractable (can be solved in $O\left(n^{2}\right)$ time) when the input graphs have bounded connected strong pathwidth.

\section{Open problems}

An interesting open problem is to find in the hierarchy depicted in Figure 9, the boundary between the parameters that give fixed parameter tractability for Graph Isomorphism, and the parameters that (probably) do not, i.e. for which Graph Isomorphism is $W[t]$ hard for some $t$ (as defined by $[8,9]$ ): until now, we only know that Graph Isomorphism 

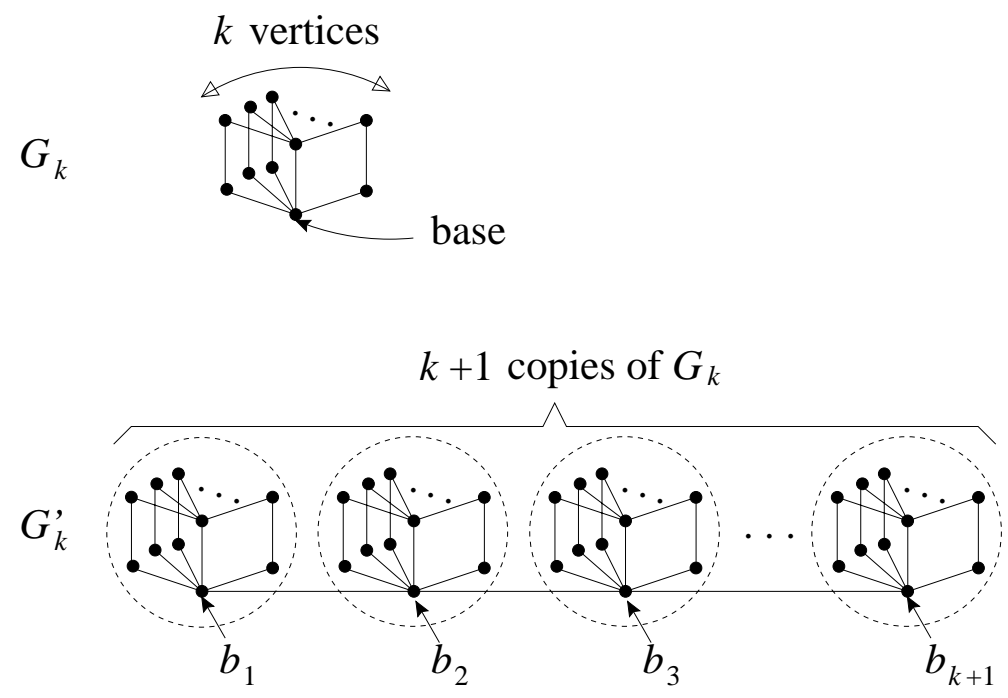

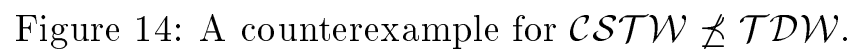

is fixed parameter tractable for parameters $\mathcal{R} \mathcal{T} \mathcal{D W}, \mathcal{R P D W}$ and $\mathcal{C S P W}$, but for all other parameters in the figure, the problem is still open.

Finally, we conjecture that, in analogy with Theorem 2.3, the problem of computing, given a graph $G$ and an integer $k$, whether $G$ has tree distance width at most $k$, is NPcomplete.

\section{References}

[1] H. L. Bodlaender, Some classes of graphs with bounded treewidth, Bulletin of the EATCS, 36 (1988), pp. 116-126.

[2] H. L. Bodlaender, A partial $k$-arboretum of graphs with bounded treewidth, Technical Report UU-CS-1996-02, Dept. of Computer Science, Utrecht University, The Netherlands.

[3] H. L. Bodlaender, Polynomial algorithms for graph isomorphism and chromatic index on partial $k$-trees, J. Algorithms, 11 (1990), pp. 631-643.

[4] F. R. K. Chung, Labelings of graphs, in Selected topics in graph theory 3 (ed. L. W. Beineke and R. J. Wilson), (1988), pp. 151-168.

[5] F. R. K. Chung and P. D. Seymour, Graphs with small bandwidth and cutwidth, Discrete Math., 75 (1989), pp. 113-119.

[6] C. J. Colbourn and K. S. Booth. Linear time automorphism algorithms for trees, interval graphs, and planar graphs. SIAM J. Comput., 10 (1981), pp. 203-225.

[7] G. Ding and B. Oporowski, Some results on tree decomposition of graphs. Journal of Graph Theory, 20 (4) (1995), pp. 481-499. 
[8] R. G. Downey and M. R. Fellows, Fixed-parameter tractability and Completeness I: Basic Results, SIAM J. Comput., 24 (1995), pp. 873-921.

[9] R. G. Downey and M. R. Fellows, Fixed-parameter tractability and completeness II: On completeness for W [1], Theor. Comput. Sci. 141 (1995), pp. 109-131.

[10] R. Halin, Tree-partitions of infinite graphs. Discrete Mathematics, 97 (1991), pp. 103-217.

[11] B. Korte, L. Lovász, H. J. Prömel and A. Schrijver ed., "Paths, Flows, and VLSILayout", Springer-Verlag (1990), pp. 383.

[12] E. M. Luks, Isomorphism of graphs of bounded valence can be tested in polynomial time, JCSS, 25 (1982), pp. 42-65.

[13] N. Robertson and P. D. Seymour, Graph Minors. X. Obstructions to TreeDecomposition, J. Combin. Theory Ser. B, 52 (1991), pp. 153-190.

[14] D. Seese, Tree-partite graphs and the complexity of algorithms, Proc. Int. Conf. on Fundamentals of Computation Theory, Lecture Notes in Computer Science 199, (1985), pp. 412-421.

[15] L. Smithline, Bandwidth of the complete $k$-ary tree, Discrete Mathematics, 142 (1995), pp. 203-212. 